\title{
Biochemical Parameters in Patients Using Teriparatide
}

\begin{abstract}
LUCRETIU RADU"\#, MARA CARSOTE2*, ANCA MIHAELA PREDESCU³, TIBERIU STEFANITA TENEA COJ AN" ${ }^{4 *}$, BOGDAN SOCEA ${ }^{5}$, VLAD DUMITRU BALEANU ${ }^{5 *}$, MIHAELA POPESCU6 ${ }^{6}$, NINA IONOVICI ${ }^{7}$, DANA MARIA ALBULESCU ${ }^{8}$

${ }^{1}$ University of Medicine and Pharmacy, Craiova, Department of Hygiene, CFR Hospital of Craiova, Stirbei Voda Str., 200374, Craiova, Romania

${ }^{2}$ Carol Davila University of Medicine and Pharmacy Department of Endocrinology, C.I.Parhon National Institute of Endocrinology, 34-38 Aviatorilor Av., 011863,Bucharest, Romania

${ }^{3}$ University of Medicine and Pharmacy of Craiova, Faculty of Dentistry Medicine, Histology Department, 2-4 Petru Rares Str., 200349, Craiova, Romania

${ }^{4}$ University of Medicine and Pharmacy of Craiova, Department of Surgery, CFR Hospital of Craiova, Stirbei-Voda Str., 200374, Craiova, Romania

${ }^{5}$ General Surgery Clinic, Emergency Clinical Hospital Sfantul Pantelimon, 340-342 Pantelimon Road, 021659, Bucharest, Romania ${ }^{6}$ University of Medicine and Pharmacy of Craiova, Department of Endocrinology, 2-4 Petru Rares Str., 200349, Craiova, Romania ${ }^{7}$ University of Medicine and Pharmacy Craiova, Department of Occupational Medicine, 2-4 Petru Rares Str.,200349, Craiova, Romania

${ }^{8}$ University of Medicine and Pharmacy Craiova, Department of Anatomy, County Hospital Craiova, 2-4 Petru Rares Str., 200349, Craiova, Romania

Teriparatide (TPT) is the active 1-34 amino acid sequence with osteoanabolic use for severe osteoporosis. Our aim is to analyze the biochemical and clinical profile of patients treated with TPT based on Romanian protocol. The inclusion and exclusion criteria are based on specific country protocol for TPT $20 \mu \mathrm{g} /$ day, for 2 years, once in life time based on self administration. This is a transversal study including data of a tertiary centre of endocrinology on patients who signed the informed consent. This is a real life study, of observational type (the intervention meaning the TPT recommendation was done by individual decision of each clinician). Normal total and ionic calcium is associated with low 25-hydroxyvitamin D levels and a mean lumbar Tscore of $-3.1 \pm 0.75 D$. 50\% of patients treated with TPT have digestive conditions, less than $10 \%$ are first time users, a high severity profile is based on a median of 4 years regarding prior anti-osteoporotic medication and of 3 previous fragility fractures.
\end{abstract}

Keywords: teriparatide, osteoporosis, calcium

Parathormone is a parathyroid gland product aiming bone health and disturbing it when primary hyperparathyroidism is registered, and also secondary parathormone raise as feedback to low vitamin D levels [1-4]. Teriparatide (TPT) is the active 1-34 section of the hormone which represents a potent osteoanabolic agent, independent of metabolic events in human body [5-9]. The indication is mainly for severe primary osteoporosis (hypogonadism- orage-related) and glucocorticoid induced bone loss which are typically seen in association with other conditions of skeleton, some with increased severity [817].

\section{Experimental part}

Aim of the study

The aim of this study is to analyse the biochemical and clinical profile of patients treated with TPT based on Romanian protocol [18].

\section{Materials and method}

This is a transversal study including data of a tertiary centre of endocrinology on patients who signed the informed consent. This is a real life study, of observational type (the intervention meaning the TPT recommendation was done by individual decision of each clinician). The inclusion and exclusion criteria of the patients are based on Romanian protocol for TPT $20 \mu \mathrm{g} /$ day, for 2 years, once in life time based on self-administration [18].

The baseline assessmentwas clinical, biochemical, and also based on central DXA (Dual X-Ray Absorptiometry), using a GE Lunar Prodigy device as mentioned in national protocol.

Data were introduced in Excel/SPSS. The parameters were expressed as mean, median, standard deviation, minimum and maximum.

\section{Results and discussions}

43 patients were included (female/male ratio was 41 / 2). Baseline parameters of age at TPT start, number of years since menopause and body mass index (BMI) are introduced in table 1. Numerous co-morbidities were identified at baseline as seen in table 2 .

The patients were introduced based on Romanian protocol: either de novo, either non-DXA responders, fracture-responders, meaning they lost BMD (Bone Mineral Density under a prior medication for osteoporosis), either non-DXA, non-fracture responders, meaning they lost BMD

\begin{tabular}{|l|c|c|c|}
\hline & Age at TPT start (yrs) & Years since menopause & BMI (KG/sqm) \\
\hline Mean & 67.093 & 21.951 & 24.569 \\
\hline SD & 8.348 & 10.151 & 4.826 \\
\hline Min & 47 & 4 & 17 \\
\hline Max & 83 & 44 & 37 \\
\hline Median & 66 & 21 & 24 \\
\hline
\end{tabular}

Table 1

STUDIED POPULATION: AGE AT TERIPARATIDE START, YEARS SINCE MENOPAUSE AND BODY MASSINDEX (BMI) 


\begin{tabular}{|l|c|}
\hline \multicolumn{1}{|c|}{ Co-morbidities } & $\mathbf{N}(\%)$ \\
\hline Digestive conditions & $20(46.51)$ \\
\hline Active corticotherapy & $4(9.30)$ \\
\hline Autoimmune conditions & $11(25.58)$ \\
\hline Arterial hypertension & $24(55.81)$ \\
\hline Hyperlipemia & $23(53.48)$ \\
\hline Chronic heart conditions & $7(16.27)$ \\
\hline Diabetes mellitus & $4(9.3)$ \\
\hline Thyroid conditions & $18(41.86)$ \\
\hline Active smokers & $6(13.95)$ \\
\hline
\end{tabular}

and had a fracture under specific medication, or DXA, nonfracture responders, as seen in table 3.

The prior number of fragility fractures and years of specific anti-osteporosis drugs exposure are introduced in table 4.

Baseline biochemistry panel is introduced in table 5 . The DXA report is centralised in table 6 . Bone turnover markers and hormones are displayed in table 7. Collateral panel of endocrine evaluation required by protocol is introduced in table 8.
Table 2

THE BASELINE CO-MORBIDITIES OF THE PATIENTS TREATED WITH TPT

Table 3

TYPES OF PATIENTS TREATED WITH TPT

\begin{tabular}{|l|c|}
\hline \multicolumn{1}{|c|}{ Type } & $\mathrm{N}(\%)$ \\
\hline De novo & $4(9.3)$ \\
\hline Non-DXA, non-fracture & $13(30.23)$ \\
\hline DXA, fracture & $10(23.25)$ \\
\hline DXA, non-fracture & $14(32.55)$ \\
\hline
\end{tabular}

\begin{tabular}{|l|c|c|c|}
\hline & Years of medication & Prior fractures & Age at first fracture \\
\hline Mean & 4.651 & 3.264 & 62.666 \\
\hline SD & 3.221 & 2.004 & 9.076 \\
\hline Min & 0 & 1 & 45 \\
\hline Max & 14 & 9 & 80 \\
\hline Median & 4 & 3 & 64 \\
\hline
\end{tabular}

Table 4

THE PRIOR NUMBER OF FRAGILITY FRACTURES AND YEARS OF SPECIFIC ANTIOSTEPOROSIS DRUGS EXPOSURE ON PATIENTS TREATED WITH TPT

Table 5

BASELINE BIOCHEMISTRY PANEL

\begin{tabular}{|l|c|c|c|c|c|}
\hline & Ionic calcium & Total calcium & 24h urinary calcium & Phosphorus & HbAlc \\
\hline Mean & 4.062 & 9.476 & 0.153 & 3.538 & 5.811 \\
\hline Sd & 0.319 & 0.352 & 0.102 & 0.69 & 0.902 \\
\hline Min & 3.1 & 8.8 & 0.03 & 2.1 & 4.8 \\
\hline Max & 4.5 & 10.2 & 0.36 & 4.8 & 8.9 \\
\hline Median & 4.1 & 9.5 & 0.12 & 3.53 & 5.6 \\
\hline Unit & $\mathrm{Mg} / \mathrm{dl}$ & $\mathrm{Mg} / \mathrm{dl}$ & $\mathrm{g} / 24 \mathrm{~h}$ & $\mathrm{Mg} / \mathrm{dl}$ & $\%$ \\
\hline Normal & $3.9-4.9$ & $8.5-10.2$ & $0.07-0.3$ & $2.5-4.5$ & $4.8-5.9$ \\
\hline
\end{tabular}

\section{Table 6}

THE DXA REPORT

\begin{tabular}{|l|c|c|c|c|c|c|c|c|}
\hline & \multicolumn{2}{|c|}{ Lumbar } & \multicolumn{2}{c|}{ Femoral neck } & \multicolumn{2}{c|}{ Total Hip } & \multicolumn{2}{c|}{ 1/3 radius } \\
\hline & BMD & T-score & BMD & T-score & BMD & T-score & BMD & T-score \\
\hline Mean & 0.806 & -3.146 & 0.706125 & -2.3725 & 0.743 & -2.09 & 0.503 & -2.908 \\
\hline SD & 0.088 & 0.727 & 0088239 & 0.69022 & 0.099 & 0.812 & 0.089 & 1.279 \\
\hline Min & 0.638 & -4.5 & 0.508 & -3.9 & 0.494 & -4.1 & 0.262 & -6.3 \\
\hline Max & 1.026 & -1.3 & 0.851 & -1.1 & 0.892 & -0.5 & 0.651 & -0.9 \\
\hline Median & 0.793 & -3.2 & 0.725 & -2.25 & 0.774 & -1.9 & 0.514 & -2.8 \\
\hline
\end{tabular}

\begin{tabular}{|l|c|c|c|c|c|c|}
\hline & AP & PINP & CL & OC & 250HD & PTH \\
\hline Mean & 71.131 & 41.841 & 0.326 & 17.169 & 29.719 & 43.743 \\
\hline SD & 22.561 & 28.208 & 0.176 & 7.725 & 13.785 & 10.918 \\
\hline Min & 45 & 13 & 0.07 & 0.32 & 6 & 24.16 \\
\hline Max & 152 & 153 & 0.885 & 43 & 58 & 65 \\
\hline Median & 65 & 31.685 & 0.28 & 16.58 & 29.7 & 43 \\
\hline Unit & U/L & $\mathrm{Ng} / \mathrm{ml}$ & $\mathrm{Ng} / \mathrm{ml}$ & $\mathrm{Ng} / \mathrm{ml}$ & $\mathrm{Ng} / \mathrm{ml}$ & $\mathrm{Pg} / \mathrm{ml}$ \\
\hline Normal & $38-105$ & $15-74$ & $0.336-1.008$ & $14-46$ & $20-100$ & $15-65$ \\
\hline
\end{tabular}

Table 7

BONE TURNOVER MARKERS AND HORMONES

$\mathrm{AP}=$ alkaline phosphatase $\mathrm{CL}=$ CrossLaps, $\mathrm{OC}=$ ostecalcin, $250 \mathrm{HD}=25$-hydroxyvitamin $\mathrm{D}, \mathrm{PTH}$ $=$ parathormone

\begin{tabular}{|l|c|c|c|c|c|}
\hline & TSH & FT4 & TPOAb & ACTH & PLASMA CORTISOL \\
\hline Mean & 1.929 & 13.517 & 31.621 & 23.263 & 13.955 \\
\hline SD & 3.072 & 2.5 & 94.333 & 9.499 & 5.663 \\
\hline Min & 0.1 & 8 & 10 & 5.6 & 5.7 \\
\hline Max & 20 & 21.6 & 577 & 44 & 26.92 \\
\hline Median & 1.1 & 13.3 & 10 & 23 & 12.955 \\
\hline Unit & $\mu$ U1/ml & $\mathrm{pmol} / \mathrm{L}$ & U/1 & $\mathrm{pg} / \mathrm{ml}$ & $\mu \mathrm{g} / \mathrm{dl}$ \\
\hline Normal & $0.5-4.5$ & $10.3-24.4$ & $<10$ & $15-65$ & $6-21$ \\
\hline
\end{tabular}

Where: TSH = Thyroid Stimulation Hormone, FT4 = Freethyroxine, TPOAb = Thyroperoxidase antibodies, $\mathrm{ACTH}=$ AdrenoCorticotropic Hormone 
The limits of the study are single centre experience and the need of follow-up data.

The strength of the study is the component of real life medicine regarding a particular protocol of an antiosteoporotic drug.

\section{Conclusions}

$50 \%$ of patients treated with PTP have digestive conditions, less than $10 \%$ are first time users, a high severity profile is based on a median of 14 years regarding prior anti-osteoporotic medication and of 3 previous fractures.

\author{
Abbreviations \\ $A P=$ alkaline phosphatase \\ $\mathrm{CL}=$ CrossLaps \\ $O C=$ ostecalcin \\ 25OHD = 25-hydroxyvitamin D \\ $\mathrm{PTH}=$ parathormone \\ $\mathrm{BMI}=$ Body Mass Index \\ $\mathrm{BTM}=$ Bone Turnover Markers \\ $\mathrm{BMD}=$ Bone Mineral Density \\ DXA = Dual-Energy X-Ray Absorptiometry \\ TPT $=$ teriparatide \\ TSH $=$ Thyroid Stimulation Hormone \\ FT4 = Freethyroxine \\ TPOAb = Thyroperoxidase antibodies \\ ACTH $=$ AdrenoCorticotropic Hormone
}

\section{References}

1.*** https://en.wikipedia.org/wiki/Parathyroid_hormone

2.POIANA C, CARSOTE M, POPESCU A, HORTOPAN D, STANESCU B, IOACHIM D, Acta Endocrinologica, III:1, 2007, p. 81.

3.GHEMIGIAN A, GHEMIGIAN M, POPESCU I, VIJA L, PETROVA E, DUMITRU N, DUMITRU I, Hormones (Athens), 12:3, 2013, p. 454460.

4.COCOLOS, A.M., DUMITRU, N., PETROVA, E.N., COCOLOS, I., TIGLIS, M., DRAGOMIRESCU, R.F.I., OLARU, M., DUMITRU, A., GHEMIGIAN, A.M., Rev. Chim. (Bucharest), 69, no. 1, 2018, p. 134.
5.MIHALACHE, L., GAVRIL, R.S., ARHIRE, L.I., NITA, O., GHERASIM, A., OPRESCU, A.C., LAPUSTE, C., CONSTANTINESCU, D., PADUREANU, S.S., Rev. Chim. (Bucharest), 67, no. 12, 2016, p. 2413-6.

6.POIANA C, RADO V, CARSOTE M, BILEZEKIAN J, Bone Research, 1:3, 2013, p. 260.

7.ARBUNE, M., LUCA ,M., MATEI, M.N., EARAR, K., ARBUNE, A., VOINESCU, D., Rev. Chim. (Bucharest), 67, no. 2, 2016, p. 320-322. 8.COLLINGE C, FAVELA J, Injury, 47:S1, 2016, p. S36-8.

9.RADU, L., CARSOTE, M., GHEORGHISAN GALATEANU, A.A., PREDA, S.A., CALBOREAN, V., STANESCU, R., GHEORMAN, V., ALBULESCU, D.M., Rev. Chim. (Bucharest), 69, no. 10, 2018 , p. 2754-8.

10.ALBULESCU, D.M., PREDA, A.S., CAMEN, A., IONOVICI, N., Rev. Chim. (Bucharest), 69, no. 7, 2018, p. 1692-4.

11.IONOVICI N, NEGRU M, GRECU D, VASILESCU M, MOGOANTA L, BOLD A, TRAISTARU R, RJ ME, 50:1, 2009, p.79-84.

12.BUGALA SA, PAVEL OR, PAVEL LP, STEFAN E, VASILE IS, ENESCU A, ENESCU AS, COVEI A, CAMEN A, IONOVICI N, ALBULESCU DM, Osteoarthritis and Musculoskeletal Diseases, 29:Suppl 1, 2018, p. 455.

13.BUGALA SA, ALBULESCU DM, IONOVICI N, CAMEN A, ENESCU A, ENESCU AS, COVEI A, VASILE IS, STEFAN E, PAVEL LP, PAVEL OR, Osteoarthritis and Musculoskeletal Diseases, 29:Suppl 1, 2018, p. 457.

14.*** https://ec.europa.eu/health/documents/community register/ 2017/20170216137058/anx 137058 ro.pdf

15.VLADU, I.M., RADU, L., GIRGAVU, S.R., TENEA COJAN, T.S., ENE, C.G., CALBOREAN, V., GHEORMAN, V., CLENCIU, D., Rev. Chim. (Bucharest), 69, no. 9, 2018, p. 2479-81.

16.ENE, C.G., ROSU, A,. GHEORMAN, V., CALBOREAN, V., TENEA COJAN, T.S., ROGOVEANU, O.C., VLADU, M.I., RADU, L, Rev. Chim. (Bucharest), 69, no. 7, 2018, p.1851-4.

17.TRAISTARU MR, KAMAL D, KAMAL KC, ROGOVEANU

OC, POPESCU M, BONDARI S, ALEXANDRU DO, IONOVICI

N, GRECU DC, Rom J Morphol Embryol, 56:4, 2015, p. 1447-53.

18.***http://amfms.ro/wp-content/uploads/2016/02/TERIPARATIDUMACIDUM ALENDRONICUM-ACIDUM-IBANDRONICUM.pdf

Manuscript received:21.07.2018 